\section{Misconceptions in interpretation of antimicrobial resistance data}

To the Editor: Recent articles ${ }^{1-3}$ indicate the increasing awareness of antimicrobial resistance and the need for antibiotic stewardship, but also illustrate misconceptions that adversely affect patient management and antibiotic prescribing.

Failure to distinguish colonisation from infection. Specimens such as urine, sputa or pus swabs, collected from non-sterile sites, are liable to contamination with the normal resident flora or with acquired colonising organisms. Pointers to genuine infection include relevant clinical symptoms and signs, pus cells on microscopy and a pure or predominant growth of a recognised pathogen on culture. Diagnosis of infection is facilitated by submitting appropriately collected specimens.

In their article on urinary tract infections among outpatient attendees in Bloemfontein, Bosch et al. ${ }^{1}$ provide no details of the type or quality of urine samples submitted and do not indicate quantitative counts, which are standard methods developed to increase accuracy in the diagnosis of urinary tract infections. No information about clinical symptoms is provided, despite asymptomatic bacteriuria requiring treatment only in certain specified circumstances, such as pregnancy or before urological surgery. ${ }^{4}$ Their data therefore provide useful information on antibiotic resistance patterns in urine samples submitted from outpatients, but not necessarily on outpatients with urinary tract infections. Similarly Truong et al. ${ }^{3}$ fail to distinguish colonisation from infection in their study on skin and soft-tissue infections with Staphylococcus aureus in Botswana.

Failure to differentiate between community and hospitalacquired infections. Major risk factors for infection or colonisation with resistant organisms are prior hospitalisation and antibiotic exposure. Attendance at certain outpatient healthcare facilities, such as dialysis units or chemotherapy units, can be included in a broader definition of healthcare-associated infections. The length of time following discharge during which a patient is still at risk of hospitalacquired infection is debatable - 2 - 3 months may be reasonable, though colonisation with organisms such as Acinetobacter baumannii may last for 6 months or more. ${ }^{5}$

In southern Africa, many chronically ill HIV-infected patients are exposed to multiple antibiotics and repeated hospitalisation for long periods, and constitute a pool of patients at high risk for infection or colonisation with resistant organisms. ${ }^{6}$ This is illustrated by Heysell et al. ${ }^{2}$ among patients hospitalised with TB in rural KwaZulu-Natal; 9/11 (82\%) patients with methicillin-resistant S. aureus (MRSA) carriage on admission had been hospitalised in the past 2 years, compared with only $17 / 41$ (41\%) of those without MRSA carriage on admission. Although MRSA carriage was not associated with HIV status, it was significantly associated with lower CD4 counts among HIV-infected patients. This finding is probably accounted for by the increased hospitalisation and antibiotic exposure in patients with advanced immunosuppression.

In the Botswana study, ${ }^{3}$ no differentiation was made between hospital-acquired and community-acquired staphylococcal isolates, but the results are not surprising when analysed according to location within the hospital. In the accident and emergency unit, which most closely approximates community-acquired infections, 62 samples cultured methicillin-sensitive $S$. aureus (MSSA), compared with only 2 samples with MRSA. Conversely, the male orthopaedic ward, which probably contains long-stay patients possibly with implanted devices, had the highest number of MRSA cultures (42) and the highest number of MSSA cultures (132).

Likewise in Bloemfontein, ${ }^{1}$ patients with positive urine cultures had high rates of recent antibiotic exposure, defined as antibiotic therapy within the previous year: $54 \%$ and $62 \%$ of uncomplicated and complicated cases respectively. Therefore the antibiotic profiles generated may not reflect those present in community-acquired infections in persons without prior antibiotic exposure.

Failure to seek microbiologist advice. Several minor technical errors also mar the articles, e.g. the reporting of multiple beta-lactam antibiotics, such as co-amoxiclav, cefotaxime and cephradine in addition to methicillin for $S$. aureus, is unnecessary as the methicillin result can be used to predict susceptibility to beta-lactam antibiotics other than penicillin. Advice from a specialist microbiologist can aid in interpreting cumulative antibiotic resistance data and the individual management of patients.

Laboratory-based surveillance can play a role in the monitoring of antibiotic resistance, but it has limitations, and medical practitioners should be wary of drawing unwarranted conclusions from such data. More valuable information can be generated through collaborative research that includes clinical information, and local microbiologists would surely be pleased to work together with clinicians in this regard.

\section{Colleen Bamford \\ NHLS \\ Cape Town \\ colleen.bamford@nhls.ac.za}

1. Bosch FJ, Van Vuuren C, Joubert G. Antimicrobial resistance patterns in outpatient urinary tract infections - the constant need to revise prescribing habits. S Afr Med J 2011;101(5):328-331.

. Heysell SK, Shenoi SV, Catterick K, Thomas TA, Friedland G. Prevalence of methicillin-resistant Staphylococcus aureus nasal carriage among hospitalised patients with tuberculosis in rural KwaZuluNatal. S Afr Med J 2011;101(5):332-334.

3. Truong H, Shah SS, Ludmir J, et al. Staphylococcus aureus skin and soft-tissue infections at a tertiary hospital in Botswana. S Afr Med J 2011;101(6):413-416.

4. Lindsay E, Bradley S, Colgan R, Rice J, Schaeffer A, Hooton T. Infectious Diseases Society of America guidelines for the diagnosis and treatment of asymptomatic bacteriuria in adults. Clin Infect Dis 2005;40:643-651.

Marchaim D, Navon-Venezia S, Schwartz D, et al. Surveillance cultures and duration of carriage of multidrug-resistant Acinetobacter baumannii. J Clin Microbiol 2007;45(5):1551-1555.

6. Cotton $\mathrm{M}$, Wasserman $\mathrm{E}$ Smit J Whitelaw A, Zar $\mathrm{H}$. High incidence of antimicrobial resistant 6.ton M, Wasserman E, Smit J, Whitelaw A, Zar H. High incidence of antimicrobial resistant organisms including extended spectrum beta-lactamase producing Enterobacteriaceae an children from Cape Town, South Africa. BMC Infect Dis 2008:8:40.

Dr Van Vuuren responds: Thank you for the observations. Antibiotic stewardship is of paramount importance for the future effective treatment of infections. Ours was a retrospective study to delineate antibiotic resistance in a specific subgroup of patients. It was therefore not possible to elicit the reasons for requesting urine culture, and this could therefore not be factored into our analysis. However, even if a patient has asymptomatic bacteruria, these data are useful to document resistance in our community.

The advantage we had was that patients received care from a single healthcare system, and a single pharmacy processed all prescriptions (even from general practitioners). It was found that an unexpectedly high percentage of patients received antibiotics for unrelated conditions in the preceding year. These antibiotics were often prescribed inappropriately for upper respiratory tract infections. This strengthens our call for antibiotic stewardship programmes not only in hospitals but also in the outpatient/primary healthcare setting.

Dr Heysell and Professor Friedland respond: We agree with the thoughtful commentary and need for greater clinician and microbiologist collaboration, particularly for rural hospitals without access to robust laboratory infrastructure. Certainly, susceptibility testing can be streamlined to relevant antibiotics, or more rapid screening methods such as that of penicillin binding protein 2a for MRSA, but must be available by means allowing prompt clinical action. As outlined in the commentary, we found MRSA carriage on 
admission to be more common in those previously hospitalised and among HIV-infected patients - those with lower CD4 counts. We acknowledged in our work the lack of definitive molecular typing of cultured isolates, and the potential variability in collection technique, and so our conclusions highlight the need for a larger-scale study to validate nosocomial acquisition. Regardless, we believe that infection control remains paramount for such a medically vulnerable population; and in many similar rural settings, simple changes can be made with significant benefit. 